\title{
Comparative characterization of two GDP- mannose dehydrogenase genes from Saccharina japonica (Laminariales, Phaeophyceae)
}

\author{
Pengyan Zhang ${ }^{1,2,3}$, Zhanru Shao ${ }^{1,2}$, Weihua $\operatorname{Jin}^{1,2}$ and Delin Duan ${ }^{1,2,4^{*}}$
}

\begin{abstract}
Background: Saccharina japonica is an important commercial brown seaweed, its main product is alginate, which is used in food, textile and by the cosmetic and pharmaceutical industries. GDP-mannose dehydrogenase (GMD) is the key enzyme involved in the synthesis of alginate. However, little is known about GMD in S. japonica. Here we report comparative biochemical analysis of two GMD genes in S. japonica.

Results: Two GMD genes from S. japonica (Sjgmd1, Sjgmd2) were cloned. The open reading frame lengths of Sjgmd1, Sjgmd2 are 963 bp and 948 bp, respectively. Alignment analysis showed that the two SjGMD sequences shared 79.38 \% identity. Both proteins possess the GGXCLPKDV and GXGXVG sequence motifs characteristic of the short-chain dehydrogenase/reductase superfamily. The optimum temperatures for SjGMDs were $30^{\circ} \mathrm{C}(\mathrm{SjGMD} 1)$ and $20^{\circ} \mathrm{C}$ (SjGMD2), and the optimum pH values were 8.0 (SjGMD1) and 8.25 (SjGMD2). Kinetic analysis demonstrated the $\mathrm{Km}$ values for the substrate GDP-mannose were $289 \mu \mathrm{M}$ (SjGMD1) and $177 \mu \mathrm{M}$ (SjGMD2), and the Km values for the cofactor $\mathrm{NAD}^{+}$were $139 \mu \mathrm{M}$ (SjGMD1) and $195 \mu \mathrm{M}$ (SjGMD2). The metal iron $\mathrm{Zn}^{2+}$ is a potent inhibitor of SjGMD1 and SjGMD2. Real-time PCR analysis showed that heat and desiccation treatments resulted in a significant increase in Sjgmd1 and Sjgmd2 transcript abundance, suggesting that the SjGMDs are directly involved in the acclimitisation of S. japonica to abiotic stresses.

Conclusion: Our work identified two novel genes encoding GMD in S. japonica, comparatively characterized their structural characteristics and enzyme kinetics, and revealed the function of GMD in the stress adaptability of S. japonica. The knowledge obtained here enriched our understanding of the alginate synthesis mechanism in S. japonica, and may promote further research on functional differences between GMD genes.
\end{abstract}

Keywords: Saccharina japonica, Alginate, GDP-mannose dehydrogenase, Enzyme kinetics, Stress response

\section{Background}

Saccharina japonica is one of the most important commercial brown seaweeds with an aquaculture production of about 5 million tons (wet weight) annually (http:// www.fao.org/fishery/statistics/en). Besides serving as food, $S$. japonica is also used for alginate production [1]. Presently nearly 30,000 tons of algin are produced annually [2].

\footnotetext{
* Correspondence: dlduan@qdio.ac.cn

${ }^{1}$ Key Laboratory of Experimental Marine Biology, Institute of Oceanology, Chinese Academy of Sciences, Qingdao 266071, China

${ }^{2}$ Laboratory for Marine Biology and Biotechnology, Qingdao National Laboratory for Marine Science and Technology, Qingdao 266071, China

Full list of author information is available at the end of the article
}

Alginate $\left(\mathrm{C}_{6} \mathrm{H}_{8} \mathrm{O}_{6}\right)_{\mathrm{n}}$ is a $\beta-1$, 4-linked linear heteropolymer consisting of variable amounts of $\beta$-D-mannuronic acid $(M)$ and its C5-epimer $\alpha$-L-guluronic acid (G) [3]. In the presence of divalent/trivalent cations, such as calcium, alginate can form algin, and its chemical properties vary with molecular weight and G/M ratio. Algin abundance and properties are different for different species [4], seasons [5], and environment conditions [1, 6, 7]. Algin possesses special gelling, viscosifying and stabilizing properties which gives it diverse applications in the food, textile, cosmetic and pharmaceutical industries. 
Alginate synthetic pathways exist in bacteria (Pseudomonas and Azotobacter) and most marine brown seaweeds [8]. The synthetic pathway of bacterial alginate has been characterized in $A$. vinelandii and $P$. aeruginosa [9, 10]. The enzymes involved, AlgA [11], AlgC [12] and AlgD [13, 14], are responsible for the synthesis of the precursor GDP-mannuronic acid, Alg8 and Alg44 are involved in the polymerization of GDP-mannuronic acid [15, 16], and AlgG epimerizes the D-mannuronate residues into L-guluronate $[2,17]$. The alginate synthetic mechanism is poorly understood in brown seaweeds. Lin et al. [18] initially proposed an alginate synthesis pathway for Fucus gardner, which was further verified in Ectocarpus siliculosus and S. japonica [19, 20]. Mannose-1-phosphate guanylyltransferase, phosphomannomutase, GDP-mannose 6-dehydrogenase (GMD), mannuronan synthase and mannuronate C5-epimerases (MC5E) have been identified in both E. siliculosus and S. japonica [19]. But so far, in brown algae, only MC5E genes from L. digitata and the GMD gene from $E$. siliculosus have been characterised biochemically $[21,22]$. There is a need to carry out a functional analysis of the alginate synthetic genes in S. japonica.

Generally, GMD has been regarded as the rate-limiting enzyme in the alginate synthesis pathway [23, 24]. It catalyzes the synthesis of GDP-mannuronic acid with the reaction: GDP-Mannose $+2 \mathrm{NAD}^{+}+\mathrm{H}_{2} \mathrm{O} \rightarrow$ GDP-Mannuronic $+2 \mathrm{NADH}$. In bacterial genomes, gmd is a single copy gene [25], while in the model brown alga E. siliculosus, it exists as a multi-copy gene, and only one gmd has been analyzed biochemically [22].

Based on our previous generated S. japonica transcriptome data [26], two GMD genes from S. japonica (Sjgmd1 and Sjgmd2) were isolated and a functional analysis was conducted. It is expected to shed light on the structures of Sjgmds and their possible roles in algal adaptation to environmental stresses, to further enrich our understanding on the alginate synthesis in brown algae.

\section{Methods}

\section{Sample collection}

S. japonica "Zhong ke No. 2" were collected from cultivated rafts in Rongcheng, Shandong, China in 2014. Sampling permission was previously received from Shandong Gaolu aquatic product Co. Ltd.. Juvenile sporophytes $(20 \sim 30 \mathrm{~cm}$ in length) in same habitat were selected as samples. The algal samples were washed with sterile seawater and precultured in darkness at $10{ }^{\circ} \mathrm{C}$ overnight. For the desiccation and heat-shock treatment, algal samples were desiccated in the darkness for $0 \mathrm{~h}, 0.5 \mathrm{~h}, 1 \mathrm{~h}, 1.5 \mathrm{~h}$. Meanwhile, other algae were cultured in darkness at $25^{\circ} \mathrm{C}$ for $0 \mathrm{~h}, 0.5 \mathrm{~h}, 1 \mathrm{~h}, 1.5 \mathrm{~h}$, respectively. All the collected samples were frozen in liquid nitrogen and stored at $-80{ }^{\circ} \mathrm{C}$.

\section{Preparation of cDNA}

Total RNA of S. japonica was extracted with RNeasy Plant Mini Kit (Qiagen, Germany) and quality was assessed using a DS-11 Spectrophotometer (Denovix, USA). High-purity RNA $\left(\mathrm{OD}_{260} / 280=1.8 \sim 2.2\right)$ were used for the synthesis of first strand cDNA according to the manual of the PrimeScript ${ }^{\mathrm{st}}$ II 1st strand cDNA synthesis kit (Takara, Dalian, China). All templates were stored at $-20{ }^{\circ} \mathrm{C}$.

\section{Isolation of SjGMD genes}

The candidate GMD unigenes were retrieved from our previous transcriptome database of $S$. japonica (GSE33853) [26], and identified by similarity analysis with the Blastx tool. To obtain the complete sequences of the GMD gene transcripts, 5' - rapid-amplification of cDNA ends (RACE) was conducted following the manual of the SMARTer RACE cDNA amplification kit (Clontech, USA), and 3'-RACE was performed using 3'Full RACE Core Set Ver.2.0 (Takara, Dalian, China). All specific primers were designed by the Primer Premier 5 software (Table 1). Based on the assembled sequence information, the open reading frames (ORF) of Sjgmd1 and Sjgmd2 were amplified with two pairs of primers (GMD1-F/GMD1-R and GMD2-F/GMD2-R) (Table 1). PrimeSTAR max DNA polymerase was used in the PCR reaction, and the amplification program was as follows: $98{ }^{\circ} \mathrm{C}$ for $5 \mathrm{~min}, 35$ cycles of $98{ }^{\circ} \mathrm{C}$ for $10 \mathrm{~s}, 55{ }^{\circ} \mathrm{C}$ for $5 \mathrm{~s}, 72{ }^{\circ} \mathrm{C}$ for $30 \mathrm{~s}$, and $72{ }^{\circ} \mathrm{C}$ for $10 \mathrm{~min}$.

\section{Bioinformatic analysis of the SjGMD genes}

Partial sequences of Sjgmd were assembled using DNAman 6.0 and the ORF was identified using the ORF finder tool (http://www.ncbi.nlm.nih.gov/gorf/gorf.html). The protein molecular weight (MW) and theoretical isoelectric point (pI) were predicted by the ProtParam [27], and the secondary structure of SjGMD1 and SjGMD2 were predicted with the SOPMA program [28]. A phylogenetic tree was constructed using the neighbor-joining algorithm in MEGA 6.0 with 1,000 bootstrap replicates [29].

\section{In vitro expression and purification of SjGMD1 and SjGMD2}

The pMAL system (New England Biolabs, USA) was used for expression and purification of fusion protein. Specific primers with NdeI and EcoRI digestion sites (GMD1-F/GMD1-R and GMD2-F/GMD2-R) were designed to amplify the ORF of Sjgmd. The purified amplification products were ligated into pMD-19T vector (Takara, Dalian, China) and digested with NdeI and EcoRI. The target bands were purified and recombined into the pMAL-c5X vector which was able to express maltose-binding protein (MBP) fusion proteins with a TEV protease cleavage site between the MBP and target protein. NEB express competent Escherichia coli were 
Table 1 List of primers used in this study

\begin{tabular}{|c|c|c|c|}
\hline Primer & Sequence ( $5^{\prime}$ to $\left.3^{\prime}\right)$ & Product size (bp) & Description \\
\hline GMD1-30 & TGGTCCACAACTACGCCAACGGGCTCAAG & 730 & 3'-RACE for Sjgmd1 \\
\hline GMD1-31 & CGACGCTCAGAAGGTGCT & & \\
\hline GMD2-30 & TTCCACCCGGACGACTGCCCGTCGGAC & 491 & 3'-RACE for Sjgmd2 \\
\hline GMD2-31 & CGACACCCGGGAGCGCATGTCCAAC & & \\
\hline GMD1-50 & GGGACTGCTCAACGAGCTTCAGCACC & 352 & 5'-RACE for Sjgmd1 \\
\hline GMD1-5I & GGCACCGAGAGGAGCACAATCTCAGG & & \\
\hline GMD1-F & CATATGATGGCTGAGGTAATGCCCAAGGAGAGC & 963 & ORF-PCR for Sjgmd 1 \\
\hline GMD1-R & GAATTCTTAGGTGGTGAGCGAGTCGGGCGA & & \\
\hline GMD2-F & CATATGATGGCCGAGCCCGAGGTGAAGAAGT & 948 & ORF-PCR for Sjgmd2 \\
\hline GMD2-R & GAATTCTTAGATGATAAGCTCGCTCGGCGAC & & \\
\hline qGMD1-F & TCCTCTCACTCTITCGGCATCC & 128 & qRT-PCR for Sjgmd 1 \\
\hline qGMD1-R & CACCCGATCTGGATGATGCTC & & \\
\hline qGMD2-F & TGCTACCTGAGCCGCAAATACG & 126 & qRT-PCR for Sjgmd2 \\
\hline qGMD2-R & CCGCCAAGAACTCCCTGAAGACC & & \\
\hline Actin-F & GACGGGTAAGGAAGAACGG & 184 & qRT-PCR for $\beta$-actin \\
\hline Actin-R & GGGACAACCAAAACAAGGGCAGGAT & & \\
\hline
\end{tabular}

used to express recombinant protein, and positive clones were collected and used for expression analysis.

Various induction temperatures $\left(15^{\circ} \mathrm{C}, 25^{\circ} \mathrm{C}, 37^{\circ} \mathrm{C}\right)$ and isopropy- $\beta$-D-thiogalactoside (IPTG) concentrations (0.1 mM, 0.3 mM, $0.5 \mathrm{mM}$ ) were applied to optimize the expression of MBP-GMD. The cell pellet was resuspended in column buffer (20 mM Tris- $\mathrm{HCl}, \mathrm{pH} 7.5$, $200 \mathrm{mM} \mathrm{NaCl}, 1 \mathrm{mM}$ ethylene diamine tetraacetic acid (EDTA), $1 \mathrm{mM} \mathrm{NaN}, 1 \mathrm{mM}$ DL-dithiothreitol (DTT), $200 \mu \mathrm{M} \mathrm{NAD}{ }^{+}$, complete protease inhibitor) and the target protein was released from the cells by sonication and purified on an amylose resin column according to the instruction manual. The purified SjGMD1 and SjGMD2 proteins were concentrated with Amiconultra15 centrifugal filter units (Millipore, MWCO $30 \mathrm{kDa}$ ) and verified using sodium dodecyl sulfate polyacrylamide gel electrophoresis (SDS-PAGE) gel (12\%).

\section{Enzyme assays}

Enzyme assays were performed by monitoring change of $\mathrm{OD}_{340}$ absorption with the Powerwave HT microplate spectrophotometer (BioTek, USA). The enzymatic assay mixture $(200 \mu \mathrm{L})$ contained $100 \mathrm{mM}$ Tris- $\mathrm{HCl}$ buffer, $0.33 \mathrm{mM}$ GDP-Mannose, $1 \mathrm{mM} \mathrm{NAD}^{+}$, and purified SjGMD protein $(30 \sim 40 \mu \mathrm{g})$. The $\mathrm{pH}$ value of Tris- $\mathrm{HCl}$ buffer was adjusted corresponding to different reaction temperature. To optimize the reaction parameter, the catalytic detections were performed under different temperatures $\left(20,25,27,30,32,35{ }^{\circ} \mathrm{C}\right.$ for SjGMD1, and 10 , $15,20,25,30{ }^{\circ} \mathrm{C}$ for $\mathrm{SjGMD} 2$ ) and different $\mathrm{pH}$ values
$(6.5,7.0,7.5,8.0,8.25,8.5,9.0,9.5)$. Each reaction was carried out for $20 \mathrm{~min}$. To calculate the $K \mathrm{~m}$ of the SjGMDs, the catalytic rates were measured under various GDP-mannose or $\mathrm{NAD}^{+}$concentrations $(0,1 / 60,1 /$ $30,1 / 15,2 / 15,4 / 15,8 / 15 \mathrm{mM}$ ) under optimal conditions and the $K \mathrm{~m}$ values were calculated by double-reciprocal plot. The effects of heavy metals were assessed by adding $1 \mathrm{mM} \mathrm{MgCl} 2, \mathrm{MnCl}_{2}, \mathrm{CaCl}_{2}$ and $\mathrm{ZnCl}_{2}$ to the standard reaction mixture. Each reaction was conducted with three replicates.

\section{GDP-mannuronic acid high performance liquid chromatography (HPLC) analysis}

One volume of sodium phosphate buffer (100 mM, $\mathrm{pH}$ 3.0) was added to terminate the enzyme assay reaction [22]. Subsequently, the mixture was centrifuged and $20 \mu \mathrm{L}$ of supernatant was injected into a HPLC system (Shimadzu-20A, Japan) with a Partisil 10 SAX column $(250 \times 4.6 \mathrm{~mm}, 10 \mu \mathrm{m}$ particle size; Whatman, USA). $10 \mathrm{mM}$ sodium phosphate $(\mathrm{pH} 3.0)$ and $750 \mathrm{mM}$ sodium phosphate $(\mathrm{pH}$ 3.7) were used as the mobile phases $\mathrm{A}$ and $B$. The separation procedure was as follows: $t_{0}$ min $3 \% \mathrm{~B} ; \mathrm{t}_{25} \min 40 \% \mathrm{~B} ; \mathrm{t}_{33} \min 75 \% \mathrm{~B} ; \mathrm{t}_{35} \min 75 \% \mathrm{~B}$; $\mathrm{t}_{56}$ min $3 \% \mathrm{~B}$ with a flow rate of $1 \mathrm{~mL} \mathrm{~min}^{-1}$ at $30{ }^{\circ} \mathrm{C}$. A ultraviolet spectrum $(230 \sim 320 \mathrm{~nm})$ was recorded using a Photo-Diode Array detector.

\section{Mass spectrometry (MS) analysis}

After the removal of the enzyme from the reaction solution by centrifugation on an Amiconultra-15 centrifugal 
filter unit (Millipore, MWCO $10 \mathrm{kDa}$ ), the solution was separated on a Sephadex G-10 gel $(1.0 \mathrm{~cm} \times 20 \mathrm{~cm})$ and eluted in water. Sugar was detected using the phenolsulfuric acid method [30], and the fraction containing sugar was selected and lyophilized.

The powder was dissolved in $\mathrm{CH}_{3} \mathrm{CN}-\mathrm{H}_{2} \mathrm{O}(1: 1, \mathrm{v} / \mathrm{v})$, and MS was performed on a LTQ ORBITRAR XL (Thermo Scientific, USA). Mass spectra were registered in the negative ion mode with a flow rate of $5 \mu \mathrm{L} \mathrm{min}{ }^{-1}$. The optimized parameters were: capillary voltage, $-3000 \mathrm{~V}$; cone voltage $-50 \mathrm{~V}$; source temperature, $80{ }^{\circ} \mathrm{C}$; dissolved temperature, $150{ }^{\circ} \mathrm{C}$. The collision energy was between 10 and $50 \mathrm{eV}$. All spectra were analyzed by Xcalibur.

Quantitative real-time PCR analysis of Sjgmd1 and Sjgmd2 Two pairs of primers (qGMD1-F/qGMD1-R, and qGMD2-F/qGMD2-R; Table 1) were designed for the amplification of $128 \mathrm{bp}$ and $126 \mathrm{bp}$ fragments, respectively. Internal control tests were conducted with the specific primers Actin-F and Actin-R [26]. The real-time PCR was performed with the SYBR Premix Ex Taq II (Takara, Dalian, China) on a TP800 Thermal Cycler Dice Real Time System (Takara, Japan). The thermal cycling protocol was: $95^{\circ} \mathrm{C}$ for $30 \mathrm{~s}$, followed by 45 cycles of $95^{\circ} \mathrm{C}$ for $5 \mathrm{~s}, 55^{\circ} \mathrm{C}$ for $10 \mathrm{~s}$ and $72{ }^{\circ} \mathrm{C}$ for $20 \mathrm{~s}$. Relative quantitative values were calculated by the $2^{-\Delta \Delta \mathrm{Ct}}$ method [31], and statistical analysis was conducted with SPSS 19.0.

\section{Results}

\section{Cloning of Sjgmd 1 and Sjgmd 2}

Four candidate unigenes from transcriptome data (Unigene50429, Unigene52620, Unigene57613, and Unigene7396) were annotated as GMD genes. On this basis, primers were designed and 352-bp and 730-bp sequences were obtained by $5^{\prime}-$ RACE and 3 '-RACE amplifications for Sjgmd1. The full-length cDNA sequence of Sjgmd1 (1523 bp, KP172530) was assembled and the length of the $5^{\prime}$-UTR, ORF and $3^{\prime}$-UTR were $84 \mathrm{bp}$, 963 bp and 476 bp, respectively. For Sjgmd2, a 491-bp sequence was obtained by 3 '-RACE amplification and a full-length cDNA sequence (1083 bp, KP172531) was assembled, which contained the 5 '-UTR (72 bp), the ORF (948 bp) and the $3^{\prime}$-UTR (63 bp).

\section{Alignment and structure analysis of SjGMDs}

Sjgmd1 encoded a protein of 320 amino acids (35.20 kDa, pI 5.22) while Sjgmd2 encoded a protein of 315 amino acids (34.46 kDa, pI 5.38). A homology analysis showed that the SjGMD proteins shared high similarity with members of the short chain dehydrogenase/ reductase (SDR) superfamily. SjGMD1 shared $91.56 \%$ identity with a GMD gene from E. siliculosus (CBJ27002), and SjGMD2 shared 93.97 \% identity with another GMD gene from E. siliculosus (CBJ29903).
However, the identity between SjGMD1 and SjGMD2 was only $79.38 \%$ with most of the variance occurring outside the conserved catalytic region. A multiple sequence alignment of GMDs showed that both SjGMDs contained the conserved NAD ${ }^{+}$-binding motif GxGxVG $(14 \sim 19$ in SjGMD1 and $11 \sim 16$ in SjGMD2) in the Nterminal region and the active motif GGxCLPKDV (259 $\sim 267$ in SjGMD1 and 256 264 in SjGMD2) in the Cterminal domain (Fig. 1). However, some key binding and catalytic residues differed between bacterial GMDs and SjGMDs, such as Val (17 in SjGMD1; 14 in SjGMD2), Ala (146 in SjGMD1; 143 in SjGMD2), Val (147 in SjGMD1; 144 in SjGMD2) and Pro (257 in SjGMD1; 254 in SjGMD2) (Fig. 1). Furthermore, as with the E. siliculosus GMDs [22] , SjGMDs contained incomplete N-terminal Rossmann folds. Predictions of secondary structure indicated that SjGMD1 contained $35.94 \%$ random coil and $36.25 \% \alpha$-helix, while SjGMD2 contained $35.24 \%$ random coil and $33.33 \% \alpha$-helix.

To understand the evolutionary history of GMD, a phylogenetic tree was constructed. All the brown algal GMDs formed a single clade, while the bacterial GMDs clustered into a separate clade (Fig. 2). In addition, the brown algal clade tended to form two separate branches, each of which included one of the SjGMDs. SjGMD1 was closer to E. siliculosus 0113 (CBJ27002) and E. siliculosus 0092 (CBJ26993), while SjGMD2 was closer to $E$. siliculosus 0053 (CBJ29903).

\section{Expression of recombinant SjGMD1 and SjGMD2}

MBP-SjGMD1 was successfully expressed after induction with $0.5 \mathrm{mM}$ IPTG for $16 \mathrm{~h}$ at $25^{\circ} \mathrm{C}$, and its MW was consistent with the predicted MW of $77.7 \mathrm{kDa}$; while MBP-SjGMD2 was induced with $0.1 \mathrm{mM}$ IPTG for $24 \mathrm{~h}$ at $15{ }^{\circ} \mathrm{C}$, and its MW was consistent with the predicted MW of $76.96 \mathrm{kDa}$ (Additional file 1). After being cleaved with factor Xa protease, the recombinant proteins were separated on SDS-PAGE (12\%), and had a MW of $35.2 \mathrm{kDa}(\mathrm{SjGMD} 1)$ and $34.46 \mathrm{kDa}$ (SjGMD2), respectively (Additional file 1). As no difference was detected between the activities of the fusion proteins and of the cleaved proteins, the recombinant SjGMD1 and SjGMD2 proteins were analysed directly in the enzymatic activity assay. The purified fusion proteins were concentrated to a final concentration of $3 \sim 4 \mathrm{mg} \mathrm{mL}^{-1}$ for the enzymatic activity assays.

\section{Enzymatic assay of SjGMD1 and SjGMD2 activities}

The optimal temperatures were $30{ }^{\circ} \mathrm{C}$ (SjGMD1) (Fig. 3a) and $20{ }^{\circ} \mathrm{C}$ (SjGMD2) (Fig. 3b), and the optimal pH values were 8.0 (SjGMD1) (Fig. 3c) and 8.25 (SjGMD2) (Fig. 3d), respectively. Since these enzymes followed the typical Michaelis-Menten kinetics model, kinetic parameters were determined. The $K \mathrm{~m}$ values of SjGMD1 were 

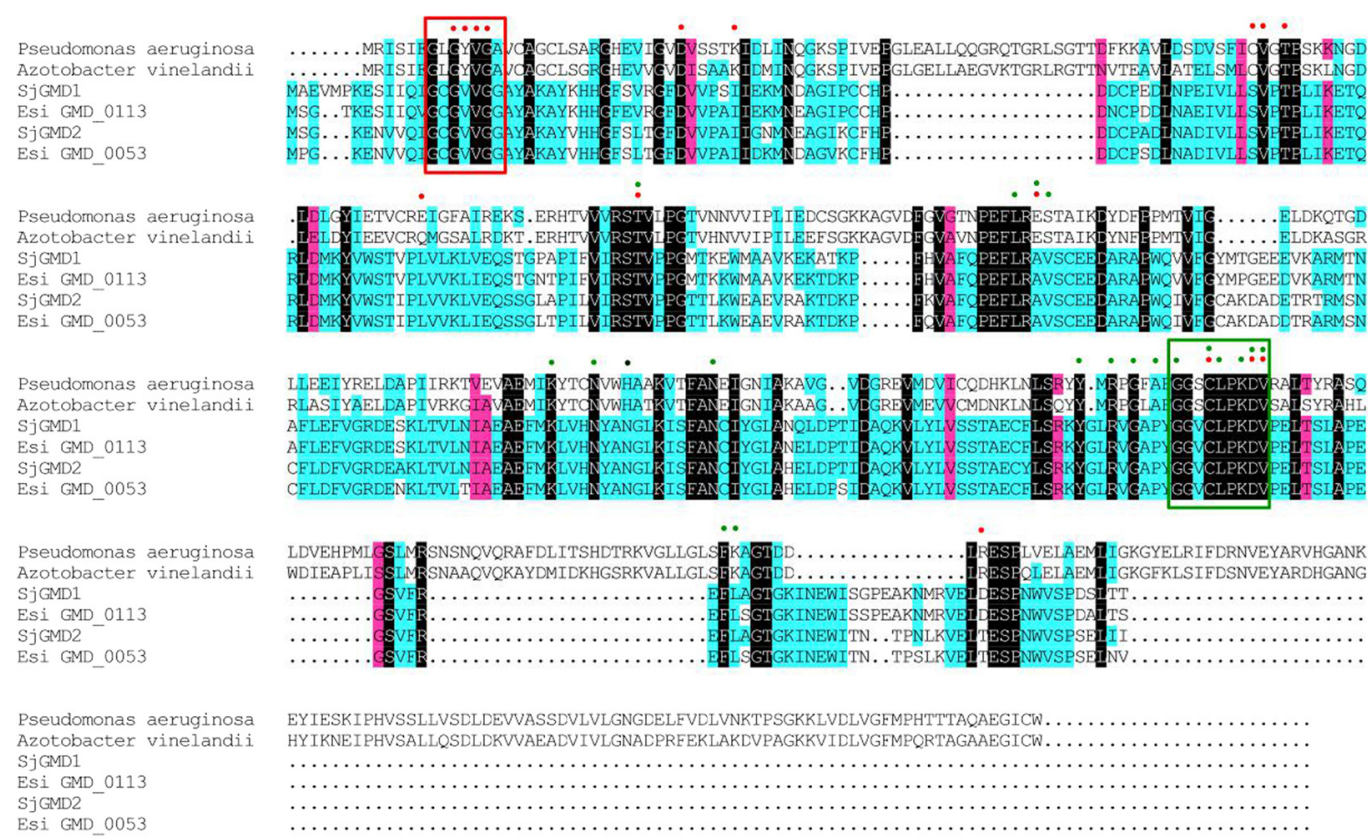

Pseudomonas aeruginosa

SjGMD1

SjGMD2
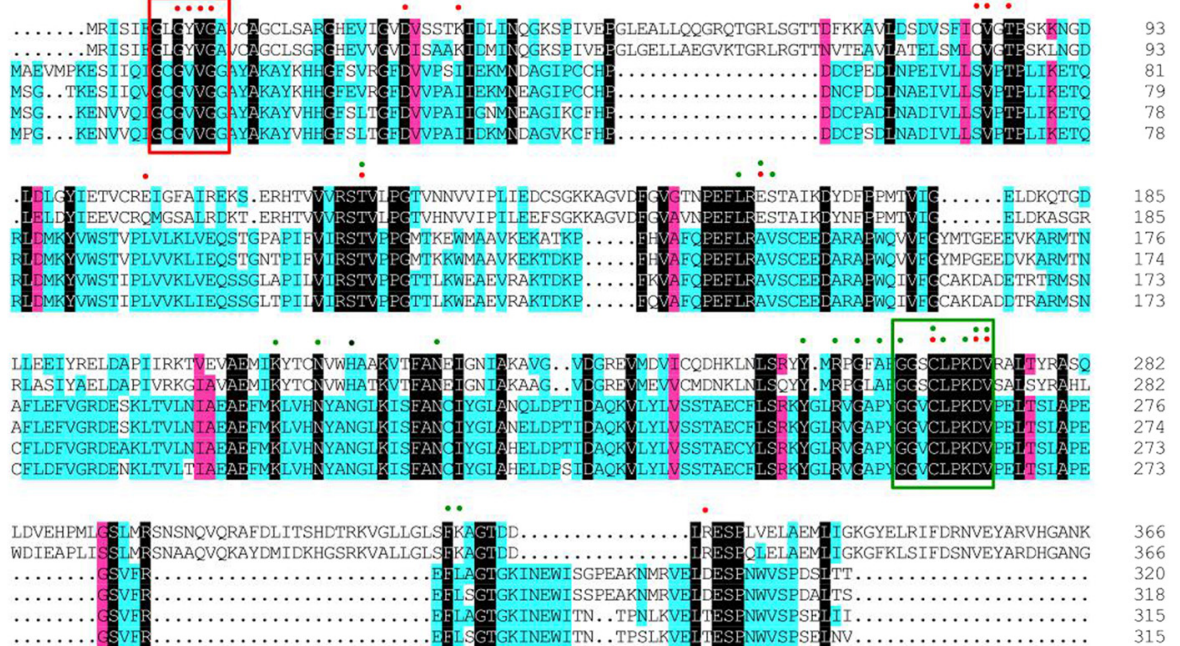

EYIESKIPHVSSLLVSDLDEVVASSDVLVLGNGDELFVDLVNKTPSGKKLVDLVGEMPHTTTAQAEGICW.

HYIKNEI PHVSALLQSDLDKVVAEADVIVLGNACPRFEKLAKDVPAGKKVIDLVGFMPQRTAGAAEGICW

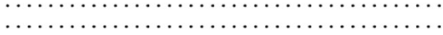

, n

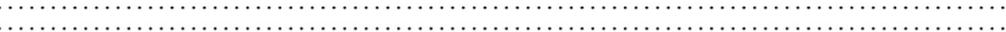

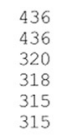

Fig. 1 Multiple sequence alignment of SjGMDs with GMDs from other species. Pseudomonas aeruginosa, WP_034074438; Azotobacter vinelandii, AAB01487; Esi GMD_0113, CBJ27002; Esi GMD_0053, CBJ29903. The conserved NAD+-binding motif GXGxVG and the active motif GGxCLPKDV are represented with red and green frames, respectively; the candidate NAD ${ }^{+}$and GDP-mannose binding sites of the SjGMDs are represented with red and green dots, respectively

$289 \mu \mathrm{M}$ for GDP-mannose (Fig. 4a) and $139 \mu \mathrm{M}$ for $\mathrm{NAD}^{+}$(Fig. 4b), while the Km values of SjGMD2 were $177 \mu \mathrm{M}$ for GDP-mannose (Fig. 4c) and $195 \mu \mathrm{M}$ for $\mathrm{NAD}^{+}$(Fig. 4d). All the applied metal ions influenced the activity of SjGMDs, among which, $\mathrm{ZnCl}_{2}$ and $\mathrm{MnCl}_{2}$ were inhibitors of SjGMDs, while $\mathrm{MgCl}_{2}$ increased the

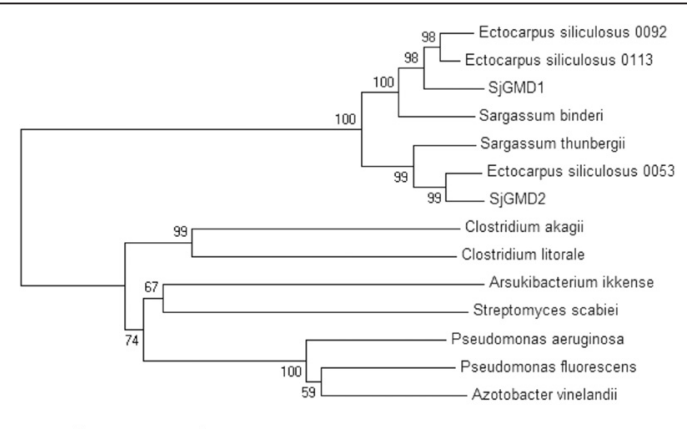

Fig. 2 Phylogenetic tree constructed based on GMD sequences. The tree was constructed using the neighbor-joining algorithm with 1,000 bootstrap replicates. Ectocarpus siliculosus 0092, CBJ26993; Ectocarpus siliculosus 0113, CBJ27002; Ectocarpus siliculosus 0053, CBJ29903; Sargassum binderi, ESTs DV668856 + DV669914; Sargassum thunbergii, SRA073158; Clostridium akagii, WP_026882540; Clostridium litorale, WP_038266433; Arsukibacterium ikkense, WP_046556127; Streptomyces scabiei, WP_013000147; Pseudomonas aeruginosa, WP_034074438; Pseudomonas fluorescens, WP_042729895; Azotobacter vinelandii, AAB01487 activity of SjGMDs (Table 2). What's more, $\mathrm{CaCl}_{2}$ acted as an activator of SjGMD1 but an inhibitor of SjGMD2 (Table 2).

\section{HPLC and MS analysis of GDP-mannuronic acid}

To confirm the catalytic activities of SjGMDs, HPLC analysis of the reaction solutions were conducted (Fig. 5). Figure $5 \mathrm{a}$ shows the peak of the substrate GDPMannose (5). Figure 5b shows that in the absence of GMD, only $\mathrm{NAD}^{+}$(1) and GDP-mannose (5) can be detected. In the following chromatograms which monitored products at reaction times of $5 \mathrm{~min}$ (Fig. $5 \mathrm{c}$ ) and 30 min (Fig. 5d), the peak areas of NADH (3) and GDPmannuronic acid (6) increased with the reaction time, while that of $\mathrm{NAD}^{+}$(1) and GDP-mannose (5) decreased significantly. This result demonstrated that GDPmannuronic acid was oxidized from GDP-mannose, and both SjGMD1 and SjGMD2 were functional.

MS analysis showed that GDP-mannuronic acid and NADH could be easily identified from the high abundance trace, consistent with the predicted mass at 618 and 664 (Fig. 6a). In addition, collision induced dissociation (CID) fragmentation of the supposed GDPmannuronic acid peak resulted in the loss of mannuronic acid or mannuronic acid without the phosphoric acid (Fig. 6b). These results further verified the production of GDP-mannuronic acid. 

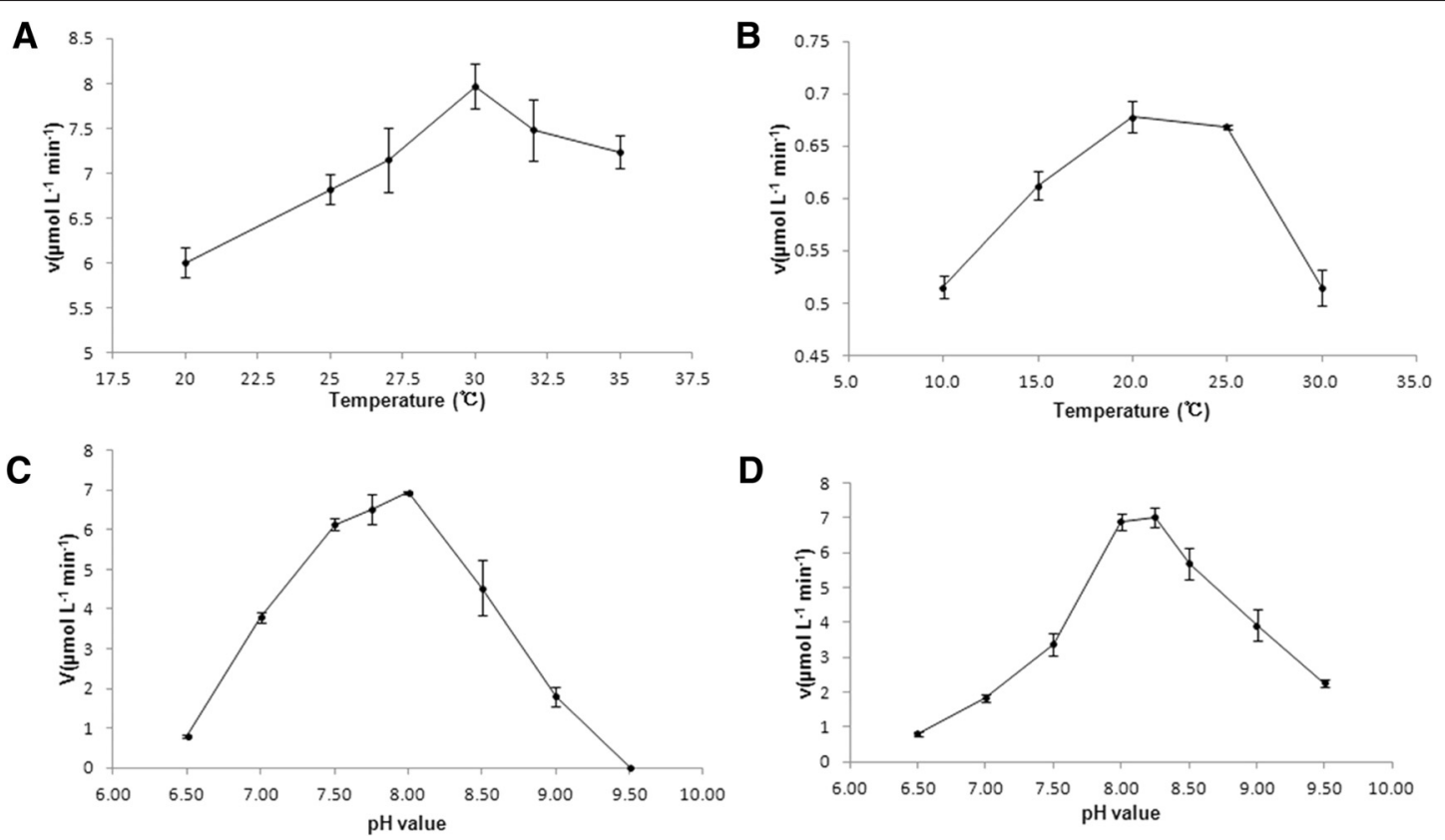

Fig. 3 Influence of temperature and pH on the activity of SjGMDs. a influence of temperature $\left(20 \sim 35^{\circ} \mathrm{C}\right)$ on the activity of SjGMD1. $\mathbf{b}$ influence of temperature $\left(10 \sim 30{ }^{\circ} \mathrm{C}\right)$ on the activity of SjGMD2. $\mathbf{c}$ influence of $\mathrm{pH}$ values $(6.5 \sim 9.5)$ on the activity of SjGMD1. $\mathbf{d}$ influence of pH values $(6.5 \sim 9.5)$ on the activity of SjGMD2

\section{Transcriptional analysis of the Sjgmds}

Under heat and desiccation stresses, Sjgmd1 and Sjgmd2 exhibited the same transcriptional profiles (Fig. 7). After heat treatment, the level of Sjgmd1 and Sjgmd2 transcripts increased significantly $(P<0.05)$ and reached a peak after
$1 \mathrm{~h}$ (5.52-fold increase for Sjgmd1 and 5.86-fold increase for Sjgmd2 compared with the control group), followed by an remarkable decrease to the original level at $1.5 \mathrm{~h}$. A similar trend was detected under desiccation stress, the level of Sjgmd1 and Sjgmd2 transcripts reached a maximum after
A

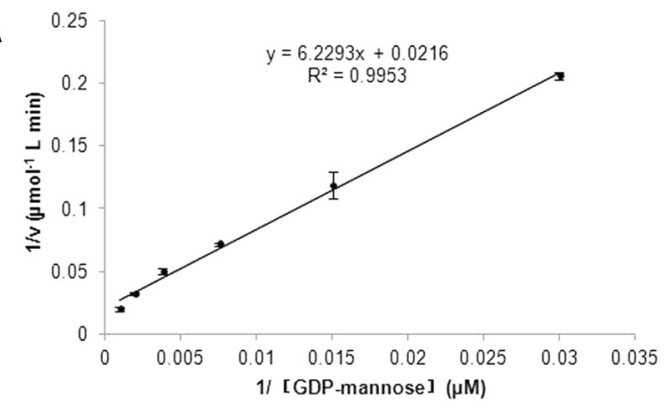

C

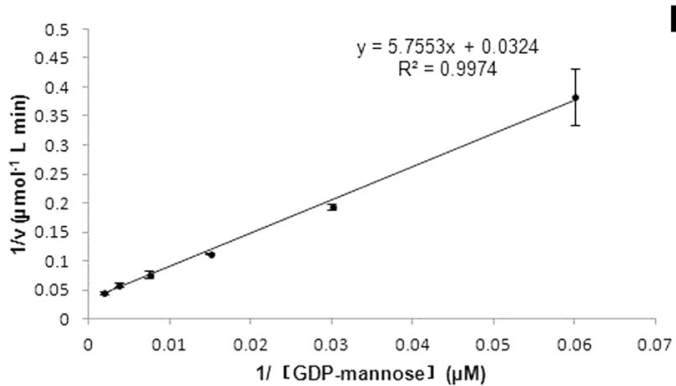

B

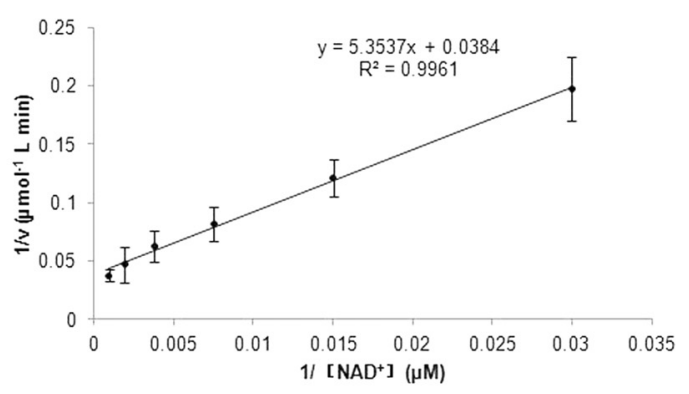

D

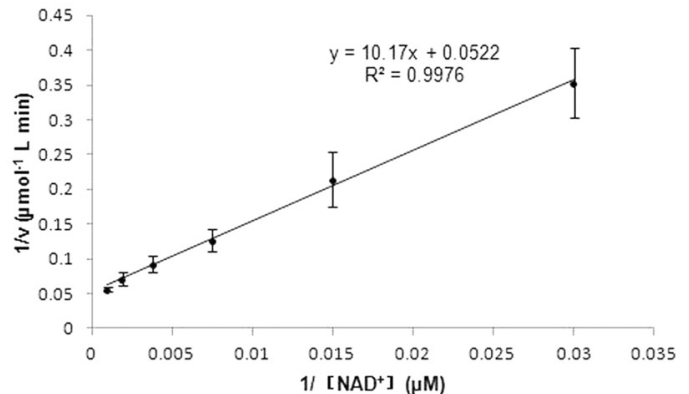

Fig. 4 Kinetic analysis of SjGMDs. a the Lineweaver-Burk plots of SjGMD1 for the substrate GDP-mannose. $\mathbf{b}$ the Lineweaver-Burk plots of SjGMD1 for the cosubstrate NAD+. $\mathbf{c}$ the Lineweaver-Burk plots of SjGMD2 for the substrate GDP-mannose. $\mathbf{d}$ the Lineweaver-Burk plots of SjGMD2 for the cosubstrate $\mathrm{NAD}^{+}$. All the values represent means $\pm \mathrm{SD}$ calculated from three reactions assays 
Table 2 Influence of metals on the enzyme activity of SjGMDs

\begin{tabular}{lcc}
\hline Metals & $\begin{array}{l}\text { Enzyme activity of SjGMD1 } \\
\text { (\% of control) }\end{array}$ & $\begin{array}{l}\text { Enzyme activity of SjGMD2 } \\
\text { (\% of control) }\end{array}$ \\
\hline control & $100.00 \pm 15.76$ & $100 \pm 13.23$ \\
$\mathrm{MgCl}_{2}$ & $133.79 \pm 16.33$ & $120.25 \pm 14.27$ \\
$\mathrm{CaCl}_{2}$ & $178.05 \pm 10.10$ & $71.86 \pm 3.87$ \\
$\mathrm{ZnCl}_{2}$ & $0.75 \pm 0.75$ & $0.75 \pm 0.24$ \\
$\mathrm{MnCl}_{2}$ & $94.01 \pm 7.43$ & $81.94 \pm 2.18$ \\
\hline
\end{tabular}

$1 \mathrm{~h}$ (110.63-fold increase for Sjgmd1 and 19.94-fold increase for Sjgmd2 compared with that of the control group).

\section{Discussion}

Two novel GMD genes from S. japonica were isolated and characterized in this study. The alignment and phylogenetic analysis showed that the variance between the two GMDs in S. japonica is higher than that between S. japonica and E. siliculosus. This indicated that the two SjGMD genes might have envolved from different GMDs rather than being derived from a duplication

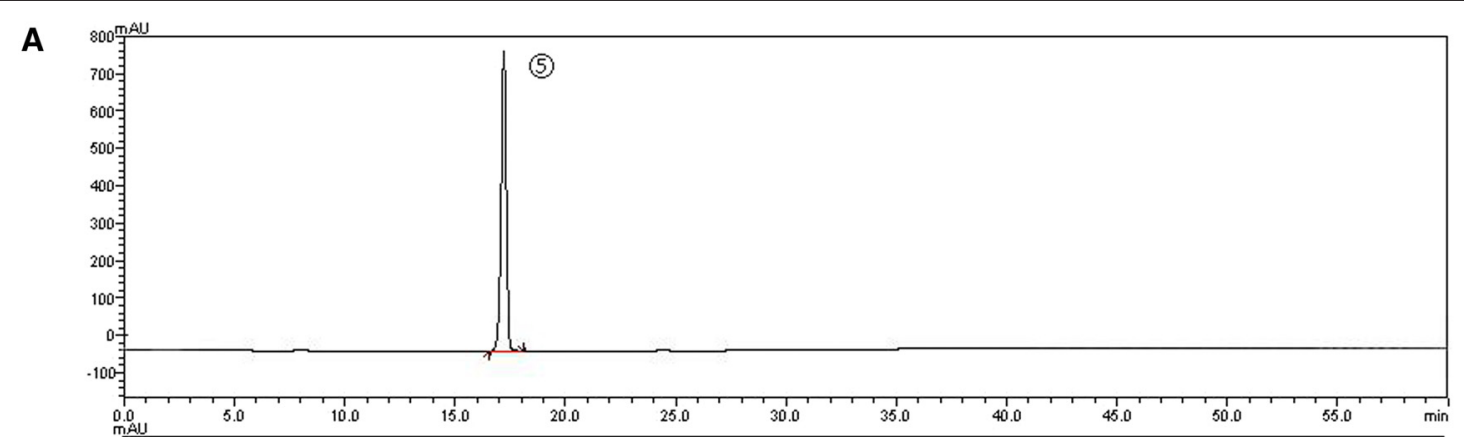

B

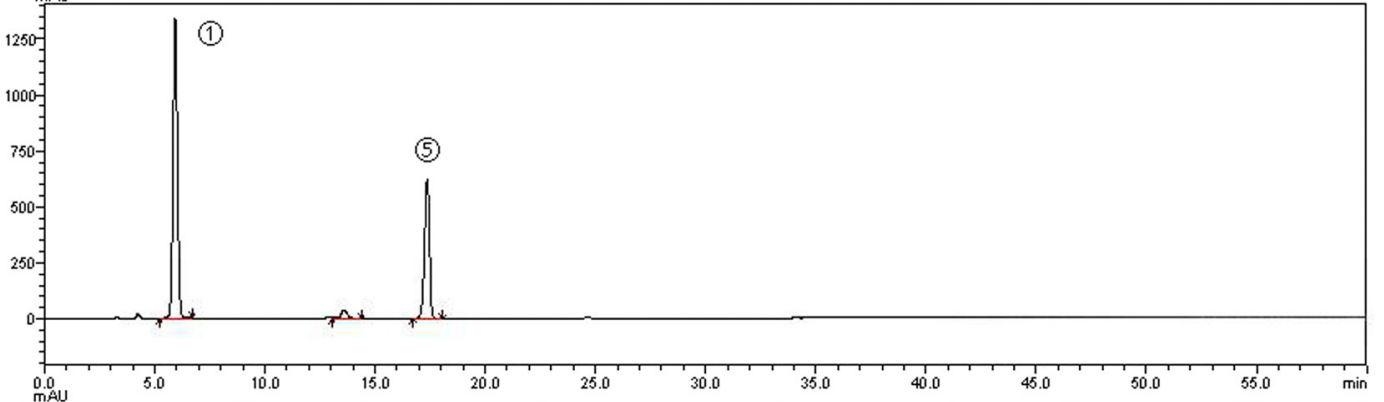

C

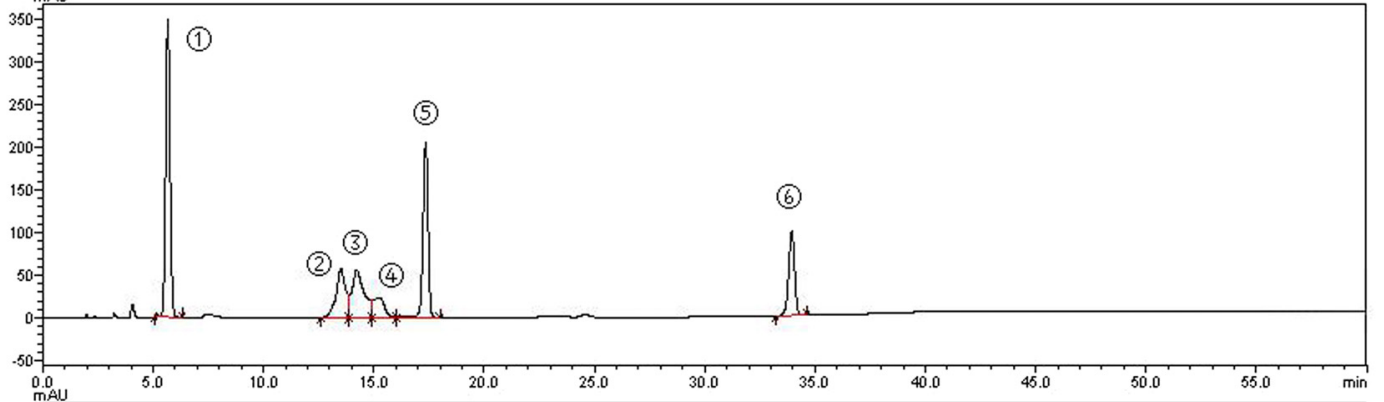

D

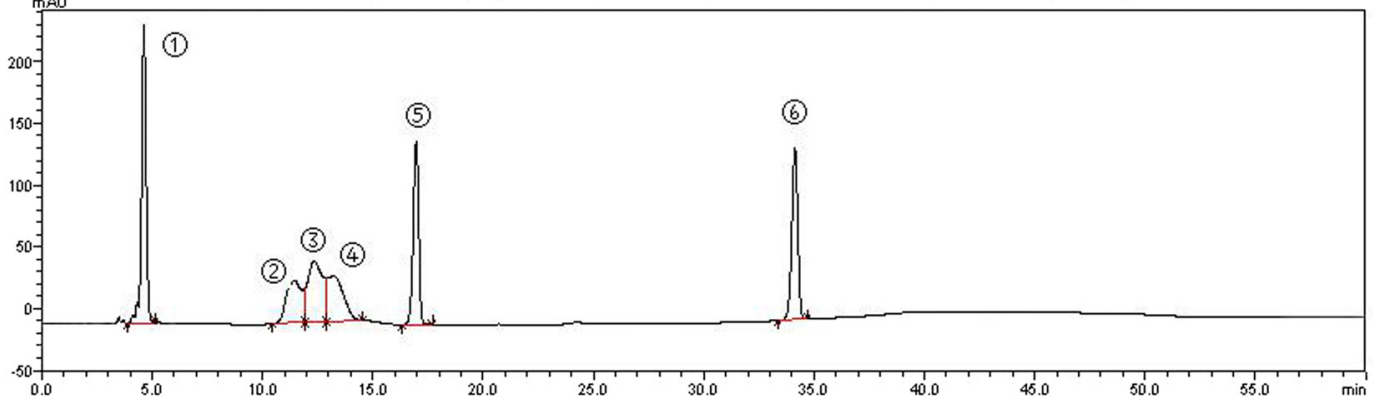

Fig. 5 HPLC chromatography of SjGMD enzyme assays. a the separation of substrate GDP-Mannose; $\mathbf{b} \sim \mathbf{d}$, the separation of enzyme assay after 0,5 and 30 min, respectively. (1), NAD ; (2) and (4), unknown impurities from NAD ${ }^{+}$; (3, NADH; (5), GDP-Mannose; (6, GDP-Mannuronic acid 


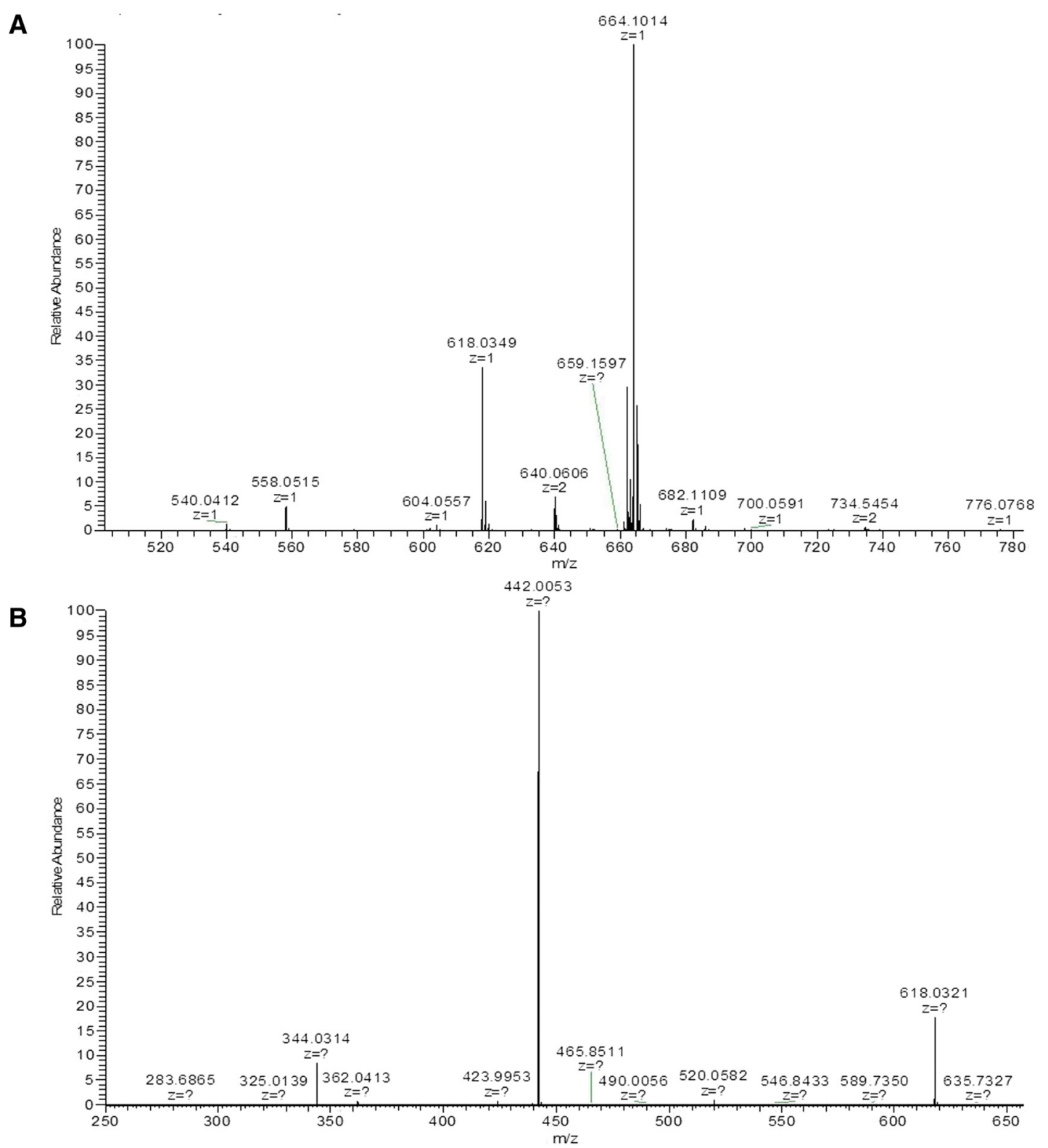

Fig. 6 MS spectrum. a negative-ion mode ESI-MS spectrum of assay mixture in a range of $500 \sim 780$. $\mathbf{b}$ negative-ion mode ESI-CID-MS/MS spectra of GDP-mannuronic acid (618.0349) in a mass range of $250 \sim 650$
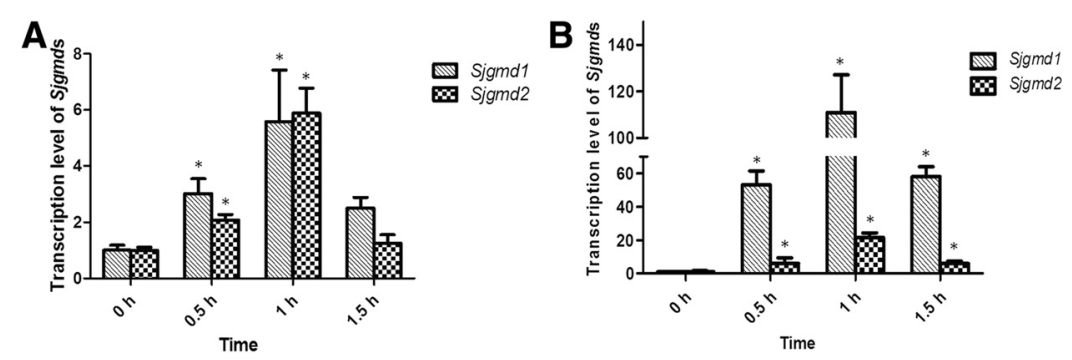

Fig. 7 Influence of heat and desiccation treatments on SjGMD genes transcription level. a expression level of Sjgmds under duration of heat stress. $\mathbf{b}$ expression level of Sjgmds under duration of desiccation stress. The treatment groups which had significant difference $(P<0.05)$ with the controls were noted. All the data are the mean values of three independent experiments 
event that happened in S. japonica. Previous research indicated that GMDs in brown algae had been gained by horizontal transfer of a single GMD from a bacterium $[19,20,22]$. Thus we proposed the GMD duplication event happened before the divergence of brown algae.

The crystal structure of GMD from $P$. aeruginosa has been reported and it showed that the enzyme contained two distinct domains connected by an R-helix and that both the $\mathrm{N}$ - and the $\mathrm{C}$-terminal domains have a typical dinucleotide-binding Rossmann fold [14]. Although the characterized SjGMDs contained conserved motifs and residues, incomplete $\mathrm{N}$-terminal Rossmann folds and residue substitutions were identified in SjGMDs, similar to the situation observed in E. siliculosus. These results suggested that brown algal GMDs may have a novel binding mechanism, suggesting distinct biochemical properties between GMDs in brown algae and in bacteria.

Though catalyzing the same reaction, the biochemical properties of SjGMD1 and SjGMD2 are quite different. The lower induction temperature indicated that SjGMD2 forms inclusion bodies more easily. The optimum temperature of $\operatorname{SjGMD} 1\left(30^{\circ} \mathrm{C}\right)$ was the same as that of $E$. siliculosus GMD [22], while that of SjGMD2 $\left(20^{\circ} \mathrm{C}\right)$ was quite different from the GMDs of both P. aeruginosa (25, 37 or $\left.50{ }^{\circ} \mathrm{C}\right)[32,33]$ and E. siliculosus $\left(30{ }^{\circ} \mathrm{C}\right)$. The optimal pHs $(8.0 ; 8.5)$ of SjGMD1 and SjGMD2 were similar to those of P. aeruginosa (7.7) and Arthrobacter sp. (8.2) $[13,34]$. This implied that the two SjGMDs exhibited different enzymatic activities. The $\mathrm{Km}$ of SjGMDs were much higher than those of bacterial GMDs [13], as which was found for E. siliculosus GMD. This may be explained by the fact that GDP-mannose is the common substrate of alginate and fucan synthesis, in that a lower $\mathrm{Km}$ might be beneficial for fucan formation [22]. Moreover, the activities of SiGMDs can be greatly affected by metal ions. $\mathrm{Zn}^{2}$ ${ }^{+}$was a strong inhibitor of both SjGMD1 and SjGMD2. This can be explained by its higher oxidability which might lead an oxidation of the catalytic residue Cys (262 in SjGMD1; 259 in SjGMD2), while $\mathrm{Mg}^{2+}$ can activate SjGMDs by improving the binding of substrate. More importantly, $\mathrm{Ca}^{2+}$ had contrasting effects on SjGMD1 and SjGMD2, which may be due to the structures of SjGMD1 and SjGMD2 undergoing different changes in configuration when bound to $\mathrm{Ca}^{2+}$. This suggested that, $\mathrm{Ca}^{2+}$ may act as a regulatory factor for SjGMD activities when the inside and outside environment of the cell change.

$S$. japonica is cold-temperate algal specie, distributed in the subtropical region of the northwest coast of the Pacific Ocean. Temperature and light are major factors that influence its growth. Desiccation can also threaten its survival. Under abiotic stresses, S. japonica can adjust its physiological and metabolic processes to acclimate and survive adverse conditions $[35,36]$. As the first line of defense, the cell wall of brown algae can thicken to increase algal tolerance to stresses [37]. Alginate is the one of the major components of the brown algal cell wall, accounting for $45 \%$ of the cell dry weight [38]. Thus, the increased alginate production might contribute to cell wall thickening. Under heat and desiccation stresses, the transcriptional level of Sjgmds increased rapidly in a short time. This result indicated that $S$. japonica increases alginate synthesis by up-regulating the expression of genes involved in the synthetic pathway. SjGMD1 and SjGMD2 expression may contribute significantly to the adaptability of S. japonica in coastal environments, to assure normal growth of alga.

\section{Conclusions}

In this article, two novel genes encoding GMD in $S$. japonica were cloned and verified. The SjGMDs exhibited sequence and structure differences and quite different biochemical properties and enzyme kinetics. The abundance of both Sjgmds transcripts increased under abiotic stresses, and this may contribute to a better adaptability of $S$. japonica. The knowledge obtained here enriched our understanding of alginate synthesis in brown algae, and provided a hint to study the functional differences between GMD genes.

\section{Availability of supporting data}

The data sets supporting the results of this article are included within the article and its additional files.

\section{Additional file}

Additional file 1: SDS-PAGE analysis of recombinant SjGMDs. (PDF 79 kb)

\section{Abbreviations}

CID: Collision induced dissociation; DTT: DL-Dithiothreitol; EDTA: Ethylene diamine tetraacetic acid; G: a-L-guluronic acid; GMD: GDP-mannose dehydrogenase; HPLC: High performance liquid chromatography; IPTG: Isopropy- $\beta$-D-thiogalactoside; M: $\beta$-D-mannuronic acid; MBP: Maltose-binding protein; MC5E: Mannuronate C5-epimerases; MS: Mass spectrometry; MW: Protein molecular weight; ORF: The open reading frame; PI: Theoretical isoelectric point; RACE: Rapid-amplification of cDNA ends; SDR superfamily: Short-chain dehydrogenase/reductase superfamily; SDS-PAGE: Sodium dodecyl sulfate polyacrylamide gel electrophoresis; Sjgmd: GDP-mannose dehydrogenase gene from S. japonica; SjGMD: GDP-mannose dehydrogenase from S. japonica.

\section{Competing interests}

The authors declare that they have no competing interests.

\section{Authors' contributions}

PYZ conducted most of the experiments, analyzed the results, and wrote the paper. ZRS made contributions to the interpretation of data and revise of the paper. WHJ designed and performed the experiments of HPLC analysis and MS analysis. DLD funded and conceived the study, and revised the paper. All authors approved the final version of the manuscript.

\section{Acknowledgments}

This work was supported by the National Key Technology Research and Development Program (2013BAB01B01) and the Ocean Public Welfare 
Scientific Research Project (201405040). We thank Dr. J. M. Cock for comments, suggestions and English revision. We also extend our sincere thanks to the anonymous reviewers for their critical comments and suggestions for the manuscript.

\section{Author details}

${ }^{1}$ Key Laboratory of Experimental Marine Biology, Institute of Oceanology, Chinese Academy of Sciences, Qingdao 266071, China. ${ }^{2}$ Laboratory for Marine Biology and Biotechnology, Qingdao National Laboratory for Marine Science and Technology, Qingdao 266071, China. ${ }^{3}$ University of Chinese Academy of Sciences, Beijing 100049, China. ${ }^{4}$ State Key Laboratory of Seaweed Bioactive Substances, Qingdao 266400, China.

\section{Received: 5 November 2015 Accepted: 27 February 2016} Published online: 08 March 2016

\section{References}

1. Bertagnolli C, Espindola APDM, Kleinübing SJ, Tasic L, da Silva MG Sargassum filipendula alginate from Brazil: Seasonal influence and characteristics. Carbohyd Polym. 2014;111:619-623.

2. Hay ID, Rehman ZU, Moradali MF, Wang YJ, Rehm BHA. Microbial alginate production, modification and its applications. Microb Biotechnol. 2013;6(6): 637-650.

3. Evans $L R$, Linker $A$. Production and characterization of the slime polysaccharide of Pseudomonas aeruginosa. J Bacteriol. 1973;116(2):915-924.

4. Haug A, Larsen B, Baardseth E. Comparison of the constitution of alginates from different sources. In: Proceedings of the VI International Seaweed Symposium. 1969. p. 443-451.

5. South GR. Alginate levels in New Zeland Durvillaea (Phaeophyceae), withparticular reference to age variations in D. antarctica. In: Proceedings of the Interna-tional Seaweed Symposium. 1979. p. 133-142.

6. Larsen B, Salem DM, Sallam MA, Mishrikey MM, Beltagy Al. Characterization of the alginates from algae harvested at the Egyptian Red Sea coast Carbohyd Res. 2003;338(22):2325-2336.

7. Davis T, Ramirez M, Mucci A, Larsen B. Extraction, isolation and cadmium binding of alginate from Sargassum spp. J Appl Phycol. 2004;16(4):275-284.

8. Draget KI, Moe ST, Skjåk-Bræk G, Smidsrød O. Alginates. In: Stephen AM, Phillips GO, Williams PA, editors. Food polysaccharides and their applications. Boca Raton: CRC Press; 2006. p. 289-334.

9. Pindar DF, Bucke C. The biosynthesis of alginic acid by Azotobacter vinelandii. J Biochem. 1975;152:617-622.

10. Piggott $\mathrm{NH}$, Sutherland IW, Jarman TR. Enzymes involved in the biosynthesis of alginate by Pseudomonas aeruginosa. European J Appl Microbiol Biotechnol. 1981:13(3):179-183.

11. May TB, Shinabarger D, Boyd A, Chakrabarty AM. Identification of amino acid residues involved in the activity of phosphomannose isomeraseguanosine 5'-diphospho-D-mannose pyrophosphorylase. A bifunctional enzyme in the alginate biosynthetic pathway of Pseudomonas aeruginosa. J Biol Chem. 1994:269(7):4872-4877.

12. Olvera C, Goldberg JB, Sánchez R, Soberón-Chávez G. The Pseudomonas aeruginosa algC gene product participates in rhamnolipid biosynthesis. FEMS Microbiol Lett. 1999;179(1):85-90.

13. Roychoudhury S, May T, Gill J, Singh S, Feingold D, Chakrabarty A. Purification and characterization of guanosine diphospho-D-mannose dehydrogenase. A key enzyme in the biosynthesis of alginate by Pseudomonas aeruginosa. J Biol Chem. 1989;264(16):9380-9385.

14. Snook CF, Tipton PA, Beamer LJ. Crystal structure of GDP-mannose dehydrogenase: a key enzyme of alginate biosynthesis in $P$. aeruginosa. Biochemistry. 2003:42(16):4658-4668.

15. Remminghorst $\mathrm{U}$, Rehm $\mathrm{BH}$. Alg44, a unique protein required for alginate biosynthesis in Pseudomonas aeruginosa. FEBS Lett. 2006;580(16):3883-3888.

16. Remminghorst $\mathrm{U}$, Rehm $\mathrm{BH}$. In vitro alginate polymerization and the functional role of Alg8 in alginate production by Pseudomonas aeruginosa. Appl environ Microb. 2006;72(1):298-305.

17. Morea A, Mathee K, Franklin MJ, Giacomini A, O'Regan M, Ohman DE. Characterization of algG encoding C5-epimerase in the alginate biosynthetic gene cluster of Pseudomonas fluorescens. Gene. 2001;278(1-2):107-114.

18. Lin T-Y, Hassid W. Pathway of alginic acid synthesis in the marine brown alga, Fucus gardneri Silva. J Biol Chem. 1966;241(22):5284-5297.

19. Michel G, Tonon T, Scornet D, Cock JM, Kloareg B. The cell wall polysaccharide metabolism of the brown alga Ectocarpus siliculosus. Insights into the evolution of extracellular matrix polysaccharides in Eukaryotes. New phytol. 2010; 188(1):82-97.

20. Ye N, Zhang X, Miao M, Fan X, Zheng Y, Xu D, et al. Saccharina genomes provide novel insight into kelp biology. Nat Commun. 2015;6:6986.

21. Nyvall P, Corre E, Boisset C, Barbeyron T, Rousvoal S, Scornet D, et al. Characterization of mannuronan C-5-epimerase genes from the brown alga Laminaria digitata. Plant physiol. 2003;133(2):726-735.

22. Tenhaken R, Voglas E, Cock JM, Neu V, Huber CG. Characterization of GDP-mannose dehydrogenase from the brown alga Ectocarpus siliculosus providing the precursor for the alginate polymer. J Biol Chem. 2011:286(19):16707-16715.

23. Deretic V, Gill JF, Chakrabartay AM. Gene algD encoding GDP-mannose dehydrogenase is transcriptionally activated in mucoid Pseudomonas aeruginosa. J Bacteriol. 1987;169:351-358.

24. Tatnell PJ, Russell NJ, Gacesa P. GDP-mannose dehydrogenase is the key regulatory enzyme in alginate biosynthesis in Pseudomonas aeruginosa: evidence from metabolite studies. Microbiology. 1994;140(7):1745-1754.

25. Chitnis CE, Ohman DE. Genetic-analysis of the alginate biosynthetic gene-cluster of Pseudomonas aeruginosa shows evidence of an operonic structure. Mol Microbiol. 1993:8(3):583-590.

26. Deng Y, Yao J, Wang X, Guo H, Duan D. Transcriptome sequencing and comparative analysis of Saccharina japonica (Laminariales, Phaeophyceae) under blue light induction. PLoS One. 2012;7(6):e39704.

27. Gasteiger E, Hoogland C, Gattiker A, Duvaud SE, Wilkins M, Appel R, et al. Protein identification and analysis tools on the ExPASy server. In: Walker J, editor. The Proteomics Protocols Handbook. New York: Humana Press; 2005. p. $571-607$

28. Geourjon C, Deléage G. SOPMA: significant improvements in protein secondary structure prediction by consensus prediction from multiple alignments. Comput Appl Biosc. 1995;11:681-684.

29. Tamura K, Stecher G, Peterson D, Filipski A, Kumar S. MEGA6: Molecular evolutionary genetics analysis version 6.0. Mol Biol Evol. 2013;30(12):2725-2729.

30. DuBois M, Gilles KA, Hamilton JK, Rebers PA, Smith F. Colorimetric method for determination of sugars and related substances. Anal Chem. 1956; 28(3):350-356.

31. Schmittgen TD, Zakrajsek BA, Mills AG, Gorn V, Singer MJ, Reed MW Quantitative reverse transcription-polymerase chain reaction to study mRNA decay: comparison of endpoint and real-time methods. Anal Biochem. 2000; 285(2):194-204.

32. Kimmel JL, Tipton PA. Inactivation of GDP-mannose dehydrogenase from Pseudomonas aeruginosa by penicillic acid identifies a critical active site loop. Arch Biochem Biophys. 2005:441(2):132-140.

33. Li F, Yu J, Yang H, Wan Z, Bai D. Effects of ambroxol on alginate of mature Pseudomonas aeruginosa biofilms. Curr Microbiol. 2008:57(1):1-7.

34. Preiss J. GDP-mannose dehydrogenase from Arthrobacter. Methods Enzymol. 1966:8:285-287.

35. Liu F, Wang W, Sun X, Liang Z, Wang F. RNA-Seq revealed complex response to heat stress on transcriptomic level in Saccharina japonica (Laminariales, Phaeophyta). J Appl Phycol. 2014;26(3):1585-1596.

36. Liu F, Wang W, Sun X, Liang Z, Wang F. Conserved and novel heat stressresponsive microRNAs were identified by deep sequencing in Saccharina japonica (Laminariales, Phaeophyta). Plant Cell Environ. 2015;38(7):13571367.

37. Fogg GE. Algal adaptation to stress-some general remarks. In: Rai L, Gaur J, editors. Algal Adaptation to Environmental Stresses. Berlin: Springer; 2001. p. 1-19.

38. Kloareg B, Demarty M, Mabeau S. Polyanionic characteristics of purified sulfated homofucans from brown-algae. Int J Biol Macromol. 1986;8:380-386 